\title{
The bounded solutions to nonlinear fifth-order differential equations with delay
}

\author{
CEMIL TUNÇ \\ Department of Mathematics, Faculty of Arts and Sciences, \\ Yüzüncü Y1l University, 65080, Van-Turkey \\ E-mail: cemtunc@yahoo.com
}

\begin{abstract}
In this paper, we improve some boundedness results, which have been obtained with respect to nonlinear differential equations of fifth order without delay, to a certain functional differential equation with constant delay. We give an illustrative example and also verify our main result by means of Liaponov tecnique.
\end{abstract}

Mathematical subject classification: $34 \mathrm{~K} 20$.

Key words: boundedness, Liapunov functional, nonlinear differential equations of fifth.

\section{Introduction}

Among the scores of articles on the qualitative theory of differential equations, the number of articles on boundedness of solutions to nonlinear fifth order differential equations with delay is significantly less than those on differential equations without delay. For those contributions on the boundedness of solutions of nonlinear fifth order differential equations without delay, one can refer to the papers of Abou-El Ela and Sadek [1], Chukwu [3], Sinha [14], Tunç [15, 16, 17], Yuan Hong [21] and some other references thereof. For those regarding the boundedness of solutions of nonlinear fifth order differential equations with delay, we cite Tunç $([18,19])$. All of the aforementioned contributions have focused on the Liapunov's second (direct) method [12] utilizing Liapunov functions and functionals. 
It is also worth mentioning that the construction of Liapunov functions and functionals for higher order nonlinear differential equations still remains as a general problem. In fact, the construction of Liapunov functional for delay differential equations of higher orders is more difficult than the derivation of Liapunov function for differential equations without delay. Since 1960 many excellent books, most of them in Russian, have been published on the qualitative behaviors of delay differential equations. See, for example, Burton [2], Èl'sgol'ts [4], Èl'sgol'ts and Norkin [5], Gopalsamy [6], Hale [7], Hale and Verduyn Lunel [8], Kolmanovskii and Myshkis [9], Kolmanovskii and Nosov [10], Krasovskii [11], Makay [13] and Yoshizawa [20] and the references listed in these books.

In this paper, we consider nonlinear fifth order delay differential equation

$$
\begin{gathered}
x^{(5)}(t)+f_{1}\left(t, x(t-r), x^{\prime}(t-r), x^{\prime \prime}(t-r), x^{\prime \prime \prime}(t-r),\right. \\
\left.x^{(4)}(t-r)\right) x^{(4)}(t)+\alpha_{2} x^{\prime \prime \prime}(t)+\alpha_{3} x^{\prime \prime}(t)+\alpha_{4} x^{\prime}(t)+f_{5}(x(t-r)) \\
=p\left(t, x(t-r), x^{\prime}(t-r), x^{\prime \prime}(t-r), x^{\prime \prime \prime}(4)(t-r)\right),
\end{gathered}
$$

which is equivalent to the system

$$
\begin{gathered}
x^{\prime}(t)=y(t), \quad y^{\prime}(t)=z(t), \quad z^{\prime}(t)=w(t), \quad w^{\prime}(t)=u(t), \\
u^{\prime}(t)=-f_{1}(t, x(t-r), y(t-r), z(t-r), w(t-r), u(t-r)) u(t) \\
\quad-\alpha_{2} w(t)-\alpha_{3} z(t)-\alpha_{4} y(t)-f_{5}(x(t))+\int_{t-r}^{t} f_{5}^{\prime}(x(s)) y(s) d s \\
\quad+p(t, x(t-r), y(t-r), z(t-r), w(t-r), u(t-r)),
\end{gathered}
$$

where $f_{1}, f_{5}$ and $p$ are continuous functions for the arguments displayed explicitly in equation (1); $r$ is a positive constant, that is, $r$ is a constant delay; $\alpha_{2}, \alpha_{3}$ and $\alpha_{4}$ are some positive constants. This fact guarantees the existence of the solution of delay differential equation (1) (see Èl'sgol'ts [4, p. 14]). It is assumed that the derivative $f_{5}^{\prime}(x) \equiv \frac{d f_{5}}{d x}$ exists and is continuous for all $x$ and all solutions considered are assumed to be real valued. In addition, we assume that the right-hand side of system (2) satisfies a Lipschitz condition in $x(t), y(t)$, $z(t), w(t), u(t), x(t-r), y(t-r), z(t-r), w(t-r)$ and $u(t-r)$. Then the solution is unique (see Èl'sgol'ts [4, p. 15]). Throughout the paper $x(t), y(t)$, 
$z(t), w(t)$ and $u(t)$ are also abbreviated as $x, y, z, w$ and $u$, respectively. It should be noted that the equation considered here, (1), is completely different than that investigated by Tunç $([18,19])$.

\section{Preliminaries}

In order to reach our main result, we will give some basic information for the general non-autonomous delay differential system, see also Burton [2], Èl'sgol'ts [4], Èl'sgol'ts and Norkin [5], Gopalsamy [6], Hale [7], Hale and Verduyn Lunel [8], Kolmanovskii and Myshkis [9], Kolmanovskii and Nosov [10], Krasovskii [11], Makay [13] and Yoshizawa [20]. Now, we consider the general non-autonomous delay differential system

$$
x^{\prime}=f\left(t, x_{t}\right), \quad x_{t}(\theta)=x(t+\theta), \quad-r \leq \theta \leq 0, \quad t \geq 0,
$$

where $f:[0, \infty) \times C_{H} \rightarrow \Re^{n}$ is a continuous mapping, $f(t, 0)=0$, and we suppose that $f$ takes closed bounded sets into bounded sets of $\Re^{n}$. Here $(C,\|\|$. is the Banach space of continuous function $\phi:[-r, 0] \rightarrow \Re^{n}$ with supremum norm, $r>0$; $C_{H}$ is the open $H$-ball in $C$;

$$
C_{H}:=\left\{\phi \in\left(C[-r, 0], \Re^{n}\right):\|\phi\|<H\right\} .
$$

Standard existence theory, see Burton [2], shows that if $\phi \in C_{H}$ and $t \geq 0$, then there is at least one continuous solution $x\left(t, t_{0}, \phi\right)$ such that on $\left[t_{0}, t_{0}+\alpha\right)$ satisfying equation (3) for $t>t_{0}, x_{t}(t, \phi)=\phi$ and $\alpha$ is a positive constant. If there is a closed subset $B \subset C_{H}$ such that the solution remains in $B$, then $\alpha=\infty$. Further, the symbol $|$.$| will denote the norm in \Re^{n}$ with $|x|=\max _{1 \leq i \leq n}\left|x_{i}\right|$.

Definition 1 (See [2]). A continuous function $W:[0, \infty) \rightarrow[0, \infty)$ with $W(0)=0, W(s)>0$ if $s>0$, and $W$ strictly increasing is a wedge. (We denote wedges by $W$ or $W_{i}$, where $i$ an integer.)

Definition 2 (See [2]). Let $D$ be an open set in $\Re^{n}$ with $0 \in D$. A function $V:[0, \infty) \times D \rightarrow[0, \infty)$ is called positive definite if $V(t, 0)=0$ and if there is a wedge $W_{1}$ with $V(t, x) \geq W_{1}(|x|)$, and is called decrescent if there is a wedge $W_{2}$ with $V(t, x) \leq W_{2}(|x|)$. 
Definition 3 (See [2]). Let $V(t, \phi)$ be a continuous functional defined for $t \geq 0, \phi \in C_{H}$. The derivative of $V$ along solutions of (3) will be denoted by $\dot{V}$ and is defined by the following relation

$$
\dot{V}(t, \phi)=\limsup _{h \rightarrow 0} \frac{V\left(t+h, x_{t+h}\left(t_{0}, \phi\right)\right)-V\left(t, x_{t}\left(t_{0}, \phi\right)\right)}{h},
$$

where $x\left(t_{0}, \phi\right)$ is the solution of (3) with $x_{t_{0}}\left(t_{0}, \phi\right)=\phi$.

Example. Let us consider the nonlinear second order delay differential equation:

$$
\begin{aligned}
x^{\prime \prime}(t)+ & \varphi\left(t, x(t-r), x^{\prime}(t-r)\right) x^{\prime}(t)+f(x(t-r)) \\
& =p\left(t, x(t), x(t-r), x^{\prime}(t), x^{\prime}(t-r)\right)
\end{aligned}
$$

which is equivalent to the system

$$
\begin{gathered}
x^{\prime}=y, \\
y^{\prime}=-\varphi(t, x(t-r), y(t-r)) y-f(x)+\int_{t-r}^{t} f^{\prime}(x(s)) y(s) d s \\
+p(t, x, x(t-r), y, y(t-r)) .
\end{gathered}
$$

We assume that the functions $\varphi$ and $p$ are continuous and satisfy the following:

$$
\varphi(t, x(t-r), y(t-r)) \geq \alpha_{1}
$$

and

$$
|p(t, x, x(t-r), y, y(t-r))| \leq q(t)
$$

for all $t, t \in[0, \infty), x, x(t-r), y$ and $y(t-r)$, where $r$ is a positive constant, constant delay, which will be determined later; $\max q(t)<\infty$ and $q \in$ $L^{1}(0, \infty) ; f$ is continuously differentiable satisfying the conditions: $x^{-1} f(x) \geq$ $\alpha_{2},(x \neq 0)$, and $\left|f^{\prime}(x)\right| \leq L$ for all $x ; \alpha_{1}, \alpha_{2}$ and $L$ are some positive constants. In particular, let us take

$$
p(t, x, x(t-r), y, y(t-r))=\frac{1}{1+t^{2}+x^{2}+x^{2}(t-r)+y^{2}+y^{2}(t-r)} .
$$


Then, it follows that

$$
\frac{1}{1+t^{2}+x^{2}+x^{2}(t-r)+y^{2}+y^{2}(t-r)} \leq \frac{1}{1+t^{2}} .
$$

If we choose

$$
q(t)=\frac{1}{1+t^{2}}
$$

then we have

$$
\int_{0}^{\infty} q(s) d s=\int_{0}^{\infty} \frac{1}{1+s^{2}} d s=\frac{\pi}{2}<\infty
$$

that is, $q \in L^{1}(0, \infty)$. Now, we introduce the Liapunov functional

$$
2 V\left(t, x_{t}, y_{t}\right)=\left[2 \int_{0}^{x} f(s) d s+y^{2}+2 \lambda \int_{-r}^{0} \int_{t+s}^{t} y^{2}(\theta) d \theta d s\right]^{\frac{1}{2}},
$$

where $\lambda$ is a positive constant which will be determined later. It is clear that the functional $V\left(t, x_{t}, y_{t}\right)$ is positive definite:

$$
\begin{gathered}
V(t, 0,0)=0 \\
V\left(t, x_{t}, y_{t}\right)=\frac{1}{2}\left[2 \int_{0}^{x} f(s) d s+y^{2}+2 \lambda \int_{-r}^{0} \int_{t+s}^{t} y^{2}(\theta) d \theta d s\right]^{\frac{1}{2}} \\
\geq \frac{1}{2}\left[2 \int_{0}^{x} \frac{f(s)}{s} s d s+y^{2}\right]^{\frac{1}{2}} \geq \frac{1}{2}\left(\alpha_{2} x^{2}+y^{2}\right)^{\frac{1}{2}}>0
\end{gathered}
$$


for all $x(\neq 0)$ and $y$. Along a trajectory of the equation, we have

$$
\begin{aligned}
\frac{d}{d t} V\left(t, x_{t}, y_{t}\right)= & \frac{1}{4}\left[2 \int_{0}^{x} f(s) d s+y^{2}+2 \lambda \int_{-r}^{0} \int_{t+s}^{t} y^{2}(\theta) d \theta d s\right]^{\frac{-1}{2}} \\
& \times\left[-2 \varphi(t, x(t-r), y(t-r)) y^{2}+2 y \int_{t-r}^{t} f^{\prime}(x(s)) y(s) d s\right. \\
& +\frac{2 y}{1+t^{2}+x^{2}+x^{2}(t-r)+y^{2}+y^{2}(t-r)} \\
& \left.+2 \lambda y^{2} r-2 \lambda \int_{t-r}^{t} y^{2}(s) d s\right] .
\end{aligned}
$$

Hence, we get

$$
\begin{gathered}
V\left(t, x_{t}, y_{t}\right) \frac{d}{d t} V\left(t, x_{t}, y_{t}\right)=-\frac{1}{4}[\varphi(t, x(t-r), y(t-r))] y^{2} \\
+\frac{1}{4} y \int_{t-r}^{t} f^{\prime}(x(s)) y(s) d s+\frac{y}{4\left(1+t^{2}+x^{2}+x^{2}(t-r)+y^{2}+y^{2}(t-r)\right)} \\
+\frac{1}{4} \lambda y^{2} r-\frac{1}{4} \lambda \int_{t-r}^{t} y^{2}(s) d s .
\end{gathered}
$$

In view of afore mentioned assumptions, the inequality $2|u v| \leq u^{2}+v^{2}$ and the fact

$$
\frac{y}{1+t^{2}+x^{2}+x^{2}(t-r)+y^{2}+y^{2}(t-r)} \leq \frac{|y|}{1+t^{2}}
$$

we find

$$
\begin{aligned}
V\left(t, x_{t}, y_{t}\right) \frac{d}{d t} V\left(t, x_{t}, y_{t}\right) \leq & -\frac{1}{8}\left[2 \alpha_{1}-(L+2 \lambda) r\right] y^{2}+\frac{|y|}{4\left(1+t^{2}\right)} \\
& +\frac{1}{8}(L-2 \lambda) \int_{t-r}^{t} y^{2}(s) d s .
\end{aligned}
$$

If we choose $\lambda=\frac{L}{2}$, then we have

$$
V\left(t, x_{t}, y_{t}\right) \frac{d}{d t} V\left(t, x_{t}, y_{t}\right) \leq-\frac{1}{4}\left[\alpha_{1}-L r\right] y^{2}+\frac{|y|}{4\left(1+t^{2}\right)} .
$$


Therefore, it follows that

$$
\begin{aligned}
V\left(t, x_{t}, y_{t}\right) \frac{d}{d t} V\left(t, x_{t}, y_{t}\right) & \leq-\frac{1}{4} \alpha y^{2}+\frac{|y|}{4\left(1+t^{2}\right)} \\
& \leq \frac{|y|}{4\left(1+t^{2}\right)} \\
& \leq \frac{1}{2\left(1+t^{2}\right)} V\left(t, x_{t}, y_{t}\right) .
\end{aligned}
$$

for some constant $\alpha>0$ provided that $r<\frac{\alpha_{1}}{L}$. Thus, we get

$$
\frac{d}{d t} V\left(t, x_{t}, y_{t}\right) \leq \frac{1}{2\left(1+t^{2}\right)} \text {. }
$$

Now, integrating this inequality from 0 to $t$ and using the fact $\frac{1}{1+t^{2}} \in$ $L^{1}(0, \infty)$, we have

$$
V\left(t, x_{t}, y_{t}\right) \leq V\left(0, x_{0}, y_{0}\right)+\frac{\pi}{4} .
$$

Therefore, one can conclude that

$$
|x| \leq K, \quad|y| \leq K
$$

for all $t \geq 0$. That is,

$$
|x| \leq K, \quad\left|x^{\prime}\right| \leq K
$$

for all $t \geq 0$. This fact shows that all solutions of the equation considered are bounded.

\section{Main result}

We establish the following result.

Theorem. In addition to the basic assumptions imposed on the functions $f_{1}$, $f_{5}$ and $p$ appearing in equation (1), we assume that there are positive constants $\alpha, \alpha_{1}, \alpha_{2}, \alpha_{3}, \alpha_{4}, \varepsilon, \varepsilon_{0}, \varepsilon_{1}, \delta$ and $\lambda$ such that the following conditions hold: 
(i) $\alpha_{1} \alpha_{2}-\alpha_{3}>0,\left(\alpha_{1} \alpha_{2}-\alpha_{3}\right) \alpha_{3}-\left(\alpha_{1} \alpha_{4}-\alpha_{5}\right) \alpha_{1}>0$,

$$
\begin{aligned}
& \delta_{0}:=\left(\alpha_{3} \alpha_{4}-\alpha_{2} \alpha_{5}\right)\left(\alpha_{1} \alpha_{2}-\alpha_{3}\right)-\left(\alpha_{1} \alpha_{4}-\alpha_{5}\right)^{2}>0 \\
& \Delta_{1}:=\frac{\left(\alpha_{3} \alpha_{4}-\alpha_{2} \alpha_{5}\right)\left(\alpha_{1} \alpha_{2}-\alpha_{3}\right)}{\alpha_{1} \alpha_{4}-\alpha_{5}}-\left(\alpha_{1} \alpha_{4}-\alpha_{5}\right) \geq 2 \varepsilon \alpha_{2} \quad \text { and } \\
& \Delta_{2}:=\frac{\alpha_{3} \alpha_{4}-\alpha_{2} \alpha_{5}}{\alpha_{1} \alpha_{4}-\alpha_{5}}-\frac{\alpha_{1} \alpha_{4}-\alpha_{5}}{\alpha_{1} \alpha_{2}-\alpha_{3}}-\frac{\varepsilon}{\alpha_{1}}>0
\end{aligned}
$$

(ii) $\varepsilon_{0} \leq f_{1}(t, x(t-r), y(t-r), z(t-r), w(t-r), u(t-r))-\alpha_{1} \leq \varepsilon_{1}$ for all $t, x(t-r), y(t-r), z(t-r), w(t-r)$ and $u(t-r)$, where the constant $\varepsilon_{1}$ satisfies the inequality

$$
\varepsilon_{1} \leq \min \left\{\frac{\varepsilon \alpha_{2}\left(\alpha_{1} \alpha_{4}-\alpha_{5}\right)^{2}}{16 \alpha_{4}^{2}\left(\alpha_{1} \alpha_{2}-\alpha_{3}\right)^{2}}, \frac{\varepsilon}{4 \alpha_{1}^{2}}, \frac{\varepsilon \alpha_{4}}{4 \delta^{2}}\right\}
$$

(iii) $f_{5}(0)=0, f_{5}(x) \neq 0$ if $x \neq 0, x^{-1} f_{5}(x) \geq \alpha(x \neq 0)$, and $f_{5}^{\prime}(x) \leq \alpha_{5}$ for all $x$.

$$
\left(f_{5}^{\prime}(x)-\alpha_{5}\right)^{2}<\min \left\{\frac{\varepsilon^{2} \alpha_{4}}{4}, \frac{\varepsilon^{2} \alpha_{2} \alpha_{4}}{16}\right\}
$$

(iv) $|p(t, x(t-r), y(t-r), z(t-r), w(t-r), u(t-r))| \leq q(t)$ for all $t$, $x(t-r), y(t-r), z(t-r), w(t-r)$ and $u(t-r)$, where $\max q(t)<\infty$ and $q \in L^{1}(0, \infty)$.

Then, there exists a finite positive constant $K$ such that the solution $x(t)$ of equation (1) defined by the initial functions

$x(t)=\phi(t), \quad x^{\prime}(t)=\phi^{\prime}(t), \quad x^{\prime \prime}(t)=\phi^{\prime \prime}(t), \quad x^{\prime \prime \prime}(t)=\phi^{\prime \prime \prime}(t), \quad t_{0}-r \leq t \leq t_{0}$

satisfies the inequalities

$$
|x(t)| \leq K, \quad\left|x^{\prime}(t)\right| \leq K, \quad\left|x^{\prime \prime}(t)\right| \leq K, \quad\left|x^{\prime \prime \prime}(t)\right| \leq K, \quad\left|x^{(4)}(t)\right| \leq K
$$

for all $t \geq t_{0}$, where $\phi \in C^{4}\left(\left[t_{0}-r, t_{0}\right], \mathfrak{R}\right)$, provided that

$$
r<\min \left\{\frac{\varepsilon_{0}}{2 \alpha_{5}}, \frac{\varepsilon}{2 \alpha_{1} \alpha_{5}}, \frac{7 \varepsilon \alpha_{2}\left(\alpha_{1} \alpha_{4}-\alpha_{5}\right)}{8 \alpha_{4} \alpha_{5}\left(\alpha_{1} \alpha_{2}-\alpha_{3}\right)}, \frac{\varepsilon \alpha_{4}}{2\left(\delta \alpha_{5}+2 \lambda\right)}\right\} \text {. }
$$


Proof. For the proof, we introduce the Liapunov functional $V_{0}=V_{0}\left(t, x_{t}, y_{t}\right.$, $\left.z_{t}, w_{t}, u_{t}\right)$ :

$$
\begin{aligned}
2 V_{0}= & u^{2}+2 \alpha_{1} u w+\frac{2 \alpha_{4}\left(\alpha_{1} \alpha_{2}-\alpha_{3}\right)}{\alpha_{1} \alpha_{4}-\alpha_{5}} u z \\
& +2 \delta u y+\left[\alpha_{1}^{2}+\alpha_{2}-\frac{\alpha_{4}\left(\alpha_{1} \alpha_{2}-\alpha_{3}\right)}{\alpha_{1} \alpha_{4}-\alpha_{5}}\right] w^{2} \\
& +2\left[\alpha_{3}+\frac{\alpha_{1} \alpha_{4}\left(\alpha_{1} \alpha_{2}-\alpha_{3}\right)}{\alpha_{1} \alpha_{4}-\alpha_{5}}-\delta\right] w z+2 \alpha_{1} \delta w y+2 \alpha_{4} y w+2 w f_{5}(x) \\
& +\alpha_{1} \alpha_{3} z^{2}+\left[\frac{\alpha_{2} \alpha_{4}\left(\alpha_{1} \alpha_{2}-\alpha_{3}\right)}{\alpha_{1} \alpha_{4}-\alpha_{5}}-\alpha_{4}-\alpha_{1} \delta\right] z^{2}+2 \delta \alpha_{2} y z+2 \alpha_{1} \alpha_{4} y z \\
& -2 \alpha_{5} z y+2 \alpha_{1} z f_{5}(x)+\frac{\alpha_{4}^{2}\left(\alpha_{1} \alpha_{2}-\alpha_{3}\right)}{\alpha_{1} \alpha_{4}-\alpha_{5}} y^{2}+\left(\delta \alpha_{3}-\alpha_{1} \alpha_{5}\right) y^{2} \\
& +\frac{2 \alpha_{4}\left(\alpha_{1} \alpha_{2}-\alpha_{3}\right)}{\alpha_{1} \alpha_{4}-\alpha_{5}} y f_{5}(x)+2 \delta \int_{0}^{x} f_{5}(\xi) d \xi+2 \lambda \int_{-r}^{0} \int_{t+s}^{t} y^{2}(\theta) d \theta d s \\
& +2 \mu \int_{-r}^{0} \int_{t+s}^{t} z^{2}(\theta) d \theta d s+2 \rho \int_{-r}^{t} \int_{t+s}^{t} w^{2}(\theta) d \theta d s,
\end{aligned}
$$

where $\lambda, \mu$ and $\rho$ are some positive constants which will be determined later in the proof and $\delta$ is also a positive constant satisfying

$$
\delta:=\frac{\alpha_{5}\left(\alpha_{1} \alpha_{2}-\alpha_{3}\right)}{\alpha_{1} \alpha_{4}-\alpha_{5}}+\varepsilon .
$$

Now, in view of (4), it follows that

$$
\begin{aligned}
2 V_{0}= & {\left[u+\alpha_{1} w+\frac{\alpha_{4}\left(\alpha_{1} \alpha_{2}-\alpha_{3}\right)}{\alpha_{1} \alpha_{4}-\alpha_{5}} z+\delta y\right]^{2}+\frac{\alpha_{4} \delta_{0}}{\left(\alpha_{1} \alpha_{4}-\alpha_{5}\right)^{2}}\left(z+\frac{\alpha_{5}}{\alpha_{4}} y\right)^{2} } \\
& +\frac{\left(\alpha_{1} \alpha_{4}-\alpha_{5}\right)}{\left(\alpha_{1} \alpha_{2}-\alpha_{3}\right)}\left[\frac{\alpha_{1} \alpha_{2}-\alpha_{3}}{\alpha_{1} \alpha_{4}-\alpha_{5}} f_{5}(x)+\frac{\alpha_{4}\left(\alpha_{1} \alpha_{2}-\alpha_{3}\right)}{\alpha_{1} \alpha_{4}-\alpha_{5}} y+\alpha_{1} z+w\right]^{2} \\
& +\Delta_{2}\left(w+\alpha_{1} z\right)^{2}+\frac{\varepsilon}{\alpha_{1}} w^{2}+2 \varepsilon\left(\frac{\alpha_{3} \alpha_{4}-\alpha_{2} \alpha_{5}}{\alpha_{1} \alpha_{4}-\alpha_{5}}\right) y z+2 \lambda \int_{-r}^{0} \int_{t+s}^{t} y^{2}(\theta) d \theta d s \\
& +2 \mu \int_{-r}^{0} \int_{t+s}^{t} z^{2}(\theta) d \theta d s+2 \rho \int_{-r}^{0} \int_{t+s}^{t} w^{2}(\theta) d \theta d s+\sum_{i=1}^{2} V_{i},
\end{aligned}
$$


where

$$
\begin{aligned}
& V_{1}:=2 \delta \int_{0}^{x} f_{5}(\xi) d \xi-\frac{\alpha_{1} \alpha_{2}-\alpha_{3}}{\alpha_{1} \alpha_{4}-\alpha_{5}} f_{5}^{2}(x), \\
& V_{2}:=\left[\delta \alpha_{3}-\alpha_{1} \alpha_{5}-\frac{\alpha_{5}^{2} \delta_{0}}{\alpha_{4}\left(\alpha_{1} \alpha_{4}-\alpha_{5}\right)^{2}}-\delta^{2}\right] y^{2} .
\end{aligned}
$$

The assumptions $f_{5}(0)=0, f_{5}(x) \operatorname{sgn} x>0, x^{-1} f_{5}(x) \geq \alpha(x \neq 0)$, $f_{5}^{\prime}(x) \leq \alpha_{5}$ and (5) imply that

$$
\begin{aligned}
V_{1} & =2 \varepsilon \int_{0}^{x} f_{5}(\xi) d \xi+\frac{2\left(\alpha_{1} \alpha_{2}-\alpha_{3}\right)}{\alpha_{1} \alpha_{4}-\alpha_{5}} \int_{0}^{x} f_{5}(\xi)\left[\alpha_{5}-f_{5}^{\prime}(\xi)\right] \\
& \geq 2 \varepsilon \int_{0}^{x} f_{5}(\xi) d \xi \geq 2 \varepsilon \int_{0}^{x} \alpha \xi d \xi=\varepsilon \alpha x^{2}
\end{aligned}
$$

and

$$
\begin{aligned}
V_{2} & =\left[\frac{\alpha_{5} \delta_{0}}{\alpha_{4}\left(\alpha_{1} \alpha_{4}-\alpha_{5}\right)}-2 \varepsilon\left(\varepsilon+\frac{2 \alpha_{5}\left(\alpha_{1} \alpha_{2}-\alpha_{3}\right)}{\alpha_{1} \alpha_{4}-\alpha_{5}}-\alpha_{3}\right)\right] y^{2} \\
& \geq \frac{\alpha_{5} \delta_{0}}{2 \alpha_{4}\left(\alpha_{1} \alpha_{4}-\alpha_{5}\right)} y^{2}
\end{aligned}
$$

provided that

$$
\frac{\alpha_{5} \delta_{0}}{4 \alpha_{4}\left(\alpha_{1} \alpha_{4}-\alpha_{5}\right)} \geq \varepsilon\left[\varepsilon+\frac{2 \alpha_{5}\left(\alpha_{1} \alpha_{2}-\alpha_{3}\right)}{\alpha_{1} \alpha_{4}-\alpha_{5}}-\alpha_{3}\right],
$$

which we now assume. Now, the estimates related to $V_{1}$ and $V_{2}$ yield

$$
\begin{aligned}
2 V_{0} \geq & {\left[u+\alpha_{1} w+\frac{\alpha_{4}\left(\alpha_{1} \alpha_{2}-\alpha_{3}\right)}{\alpha_{1} \alpha_{4}-\alpha_{5}} z+\delta y\right]^{2} } \\
& +\frac{\alpha_{4} \delta_{0}}{\left(\alpha_{1} \alpha_{4}-\alpha_{5}\right)^{2}}\left(z+\frac{\alpha_{5}}{\alpha_{4}} y\right)^{2}+\varepsilon \alpha x^{2}+\Delta_{2}\left(w+\alpha_{1} z\right)^{2} \\
& +\frac{\alpha_{5} \delta_{0}}{2 \alpha_{4}\left(\alpha_{1} \alpha_{4}-\alpha_{5}\right)} y^{2} \frac{\varepsilon}{\alpha_{1}} w^{2}+2 \varepsilon\left(\frac{\alpha_{3} \alpha_{4}-\alpha_{2} \alpha_{5}}{\alpha_{1} \alpha_{4}-\alpha_{5}}\right) y z \\
& +2 \lambda \int_{-r}^{0} \int_{t+s}^{t} y^{2}(\theta) d \theta d s+2 \mu \int_{t+s}^{t} z^{2}(\theta) d \theta d s \\
& +2 \rho \int_{-r}^{t} \int_{t+s}^{t} w^{2}(\theta) d \theta d s .
\end{aligned}
$$


Following a similar way as that of Tunç [18], one can easily conclude from (6) that

$$
V_{0} \geq D_{8}\left(x^{2}+y^{2}+z^{2}+w^{2}+u^{2}\right)
$$

for a sufficiently small positive constant $D_{8}$. Now, let

$$
\frac{d}{d t} V_{0}\left(t, x_{t}, y_{t}, z_{t}, w_{t}, u_{t}\right)=\dot{V}_{0} \quad \text { and } \quad(x(t), y(t), z(t), w(t), u(t))
$$

be a solution of system (2). Then, a direct computation along this solution shows that

$$
\begin{aligned}
\frac{d}{d t} & V_{0}\left(t, x_{t}, y_{t}, z_{t}, w_{t}, u_{t}\right)= \\
& -\left[f_{1}(t, x(t-r), y(t-r), z(t-r), w(t-r), u(t-r))-\alpha_{1}\right] u^{2} \\
& -\left[\alpha_{1} \alpha_{2}-\left\{\alpha_{3}+\frac{\alpha_{1} \alpha_{4}\left(\alpha_{1} \alpha_{2}-\alpha_{3}\right)}{\alpha_{1} \alpha_{4}-\alpha_{5}}-\delta\right\}\right] w^{2} \\
& -\left[\frac{\alpha_{3} \alpha_{4}\left(\alpha_{1} \alpha_{2}-\alpha_{3}\right)}{\alpha_{1} \alpha_{4}-\alpha_{5}}-\left\{\delta \alpha_{2}+\alpha_{1} \alpha_{4}-\alpha_{5}\right\}\right] z^{2}-\left[\delta \alpha_{4}-\frac{\alpha_{4}\left(\alpha_{1} \alpha_{2}-\alpha_{3}\right)}{\alpha_{1} \alpha_{4}-\alpha_{5}} f_{5}^{\prime}(x)\right] y^{2} \\
& -\alpha_{1}\left[f_{1}(t, x(t-r), y(t-r), z(t-r), w(t-r), u(t-r))-\alpha_{1}\right] w u \\
& -\frac{\alpha_{4}\left(\alpha_{1} \alpha_{2}-\alpha_{3}\right)}{\alpha_{1} \alpha_{4}-\alpha_{5}}\left[f_{1}(t, x(t-r), y(t-r), z(t-r), w(t-r), u(t-r))-\alpha_{1}\right] z u \\
& -\delta\left[f_{1}(t, x(t-r), y(t-r), z(t-r), w(t-r), u(t-r))-\alpha_{1}\right] y u \\
& -\left[\alpha_{5}-f_{5}^{\prime}(x)\right] y w-\alpha_{1}\left[\alpha_{5}-f_{5}^{\prime}(x)\right] y z+u \int_{t-r}^{t} f_{5}^{\prime}(x(s)) y(s) d s \\
& +\alpha_{1} w \int_{t-r}^{t} f_{5}^{\prime}(x(s)) y(s) d s+\frac{\alpha_{4}\left(\alpha_{1} \alpha_{2}-\alpha_{3}\right)}{\alpha_{1} \alpha_{4}-\alpha_{5}} z \int_{t-r}^{t} f_{5}^{\prime}(x(s)) y(s) d s \\
& +\delta y \int_{t-r}^{t} f_{5}^{\prime}(x(s)) y(s) d s+\left[u+\alpha_{1} w+\frac{\alpha_{4}\left(\alpha_{1} \alpha_{2}-\alpha_{3}\right)}{\alpha_{1} \alpha_{4}-\alpha_{5}} z+\delta y\right] \\
& \times p(t, x(t-r), y(t-r), z(t-r), w(t-r), u(t-r)) \\
& +\lambda y^{2} r-\lambda \int_{t-r}^{t} y^{2}(s) d s+\mu z^{2} r-\mu d s+\rho w^{2} r-\rho
\end{aligned}
$$


In view of the assumptions of theorem and expression (5), we have that

$$
\begin{aligned}
& {\left[\alpha_{1} \alpha_{2}-\left\{\alpha_{3}+\frac{\alpha_{1} \alpha_{4}\left(\alpha_{1} \alpha_{2}-\alpha_{3}\right)}{\alpha_{1} \alpha_{4}-\alpha_{5}}-\delta\right\}\right] \geq \varepsilon,} \\
& \frac{\alpha_{3} \alpha_{4}\left(\alpha_{1} \alpha_{2}-\alpha_{3}\right)}{\alpha_{1} \alpha_{4}-\alpha_{5}}-\left(\delta \alpha_{2}+\alpha_{1} \alpha_{4}-\alpha_{5}\right)>\varepsilon \alpha_{2}
\end{aligned}
$$

and

$$
\delta \alpha_{4}-\frac{\alpha_{4}\left(\alpha_{1} \alpha_{2}-\alpha_{3}\right)}{\alpha_{1} \alpha_{4}-\alpha_{5}} f_{5}^{\prime}(x) \geq \varepsilon \alpha_{4} .
$$

By the assumption $f_{5}^{\prime}(x) \leq \alpha_{5}$ and inequality $2|u v| \leq u^{2}+v^{2}$, we obtain the following:

$$
\begin{aligned}
& u \int_{t-r}^{t} f_{5}^{\prime}(x(s)) y(s) d s \leq \frac{\alpha_{5}}{2} r u^{2}+\frac{\alpha_{5}}{2} \int_{t-r}^{t} y^{2}(s) d s, \\
& \alpha_{1} w \int_{t-r}^{t} f_{5}^{\prime}(x(s)) y(s) d s \leq \frac{\alpha_{1} \alpha_{5}}{2} r w^{2}+\frac{\alpha_{1} \alpha_{5}}{2} \int_{t-r}^{t} y^{2}(s) d s, \\
& \frac{\alpha_{4}\left(\alpha_{1} \alpha_{2}-\alpha_{3}\right)}{\alpha_{1} \alpha_{4}-\alpha_{5}} z \int_{t-r}^{t} f_{5}^{\prime}(x(s)) y(s) d s \leq \frac{\alpha_{4} \alpha_{5}\left(\alpha_{1} \alpha_{2}-\alpha_{3}\right)}{2\left(\alpha_{1} \alpha_{4}-\alpha_{5}\right)} r z^{2} \\
& \quad+\frac{\alpha_{4} \alpha_{5}\left(\alpha_{1} \alpha_{2}-\alpha_{3}\right)}{2\left(\alpha_{1} \alpha_{4}-\alpha_{5}\right)} \int_{t-r}^{t} y^{2}(s) d s
\end{aligned}
$$

and

$$
\delta y \int_{t-r}^{t} f_{5}^{\prime}(x(s)) y(s) d s \leq \frac{\delta \alpha_{5}}{2} r y^{2}+\frac{\delta \alpha_{5}}{2} \int_{t-r}^{t} y^{2}(s) d s .
$$

Making use of these inequalities, we get

$$
\begin{aligned}
\dot{V}_{0} & \leq-\left[f_{1}(t, x(t-r), y(t-r), z(t-r), w(t-r), u(t-r))-\alpha_{1}\right] u^{2} \\
& -\varepsilon w^{2}-\left(\varepsilon \alpha_{2}\right) z^{2}-\left(\varepsilon \alpha_{4}\right) y^{2} \\
& -\alpha_{1}\left[f_{1}(t, x(t-r), y(t-r), z(t-r), w(t-r), u(t-r))-\alpha_{1}\right] w u \\
& -\frac{\alpha_{4}\left(\alpha_{1} \alpha_{2}-\alpha_{3}\right)}{\alpha_{1} \alpha_{4}-\alpha_{5}}\left[f_{1}(t, x(t-r), y(t-r), z(t-r), w(t-r), u(t-r))-\alpha_{1}\right] z u \\
& -\delta\left[f_{1}(t, x(t-r), y(t-r), z(t-r), w(t-r), u(t-r))-\alpha_{1}\right] y u
\end{aligned}
$$




$$
\begin{aligned}
& +\left[f_{5}^{\prime}(x)-\alpha_{5}\right] y w+\alpha_{1}\left[f_{5}^{\prime}(x)-\alpha_{5}\right] y z+\left|u+\alpha_{1} w+\frac{\alpha_{4}\left(\alpha_{1} \alpha_{2}-\alpha_{3}\right)}{\alpha_{1} \alpha_{4}-\alpha_{5}} z+\delta y\right| \\
& \times|p(t, x(t-r), y(t-r), z(t-r), w(t-r), u(t-r))| \\
& +\frac{\alpha_{5}}{2} r u^{2}+\frac{\alpha_{1} \alpha_{5}}{2} r w^{2}+\frac{\alpha_{4} \alpha_{5}\left(\alpha_{1} \alpha_{2}-\alpha_{3}\right)}{2\left(\alpha_{1} \alpha_{4}-\alpha_{5}\right)} r z^{2}+\frac{\delta \alpha_{5}}{2} r y^{2} \\
& +\lambda r y^{2}+\mu z^{2} r+\rho w^{2} r+\mu \int_{t-r}^{t} z^{2}(s) d s-\rho \int_{t-r}^{t} w^{2}(s) d s \\
& +\left[\frac{\alpha_{4} \alpha_{5}\left(\alpha_{1} \alpha_{2}-\alpha_{3}\right)}{2\left(\alpha_{1} \alpha_{4}-\alpha_{5}\right)}+\frac{\alpha_{5}}{2}+\frac{\alpha_{1} \alpha_{5}}{2}+\frac{\delta \alpha_{5}}{2}-\lambda\right] \int_{t-r}^{t} y^{2}(s) d s \\
& \leq-\frac{1}{4}\left[\left(f_{1}(t, x(t-r), y(t-r), z(t-r), w(t-r), u(t-r))-\alpha_{1}\right)-2 \alpha_{5} r\right] u^{2} \\
& -\frac{1}{4}\left[2 \varepsilon-\left(\rho+\frac{\alpha_{1} \alpha_{5}}{2}\right) r\right] w^{2}-\left[\frac{7 \varepsilon \alpha_{2}}{8}-\left(\mu+\frac{\alpha_{4} \alpha_{5}\left(\alpha_{1} \alpha_{2}-\alpha_{3}\right)}{2\left(\alpha_{1} \alpha_{4}-\alpha_{5}\right)}\right) r\right] z^{2} \\
& -\left[\frac{\varepsilon \alpha_{4}}{4}-\left(\frac{\delta \alpha_{5}}{2}+\lambda\right) r\right] y^{2}+\left|u+\alpha_{1} w+\frac{\alpha_{4}\left(\alpha_{1} \alpha_{2}-\alpha_{3}\right)}{\alpha_{1} \alpha_{4}-\alpha_{5}} z+\delta y\right| \\
& \times|p(t, x(t-r), y(t-r), z(t-r), w(t-r), u(t-r))| \\
& +\left[\frac{\alpha_{4} \alpha_{5}\left(\alpha_{1} \alpha_{2}-\alpha_{3}\right)}{2\left(\alpha_{1} \alpha_{4}-\alpha_{5}\right)}+\frac{\alpha_{5}}{2}+\frac{\alpha_{1} \alpha_{5}}{2}+\frac{\delta \alpha_{5}}{2}-\lambda\right] \int_{t-r}^{t} y^{2}(s) d s \\
& -\mu \int_{t-r}^{t} z^{2}(s) d s-\rho \int_{t-r}^{t} w^{2}(s) d s-\sum_{k=4}^{8} V_{k}
\end{aligned}
$$

where

$$
\begin{aligned}
V_{4}= & \frac{1}{4}\left[f_{1}(t, x(t-r), y(t-r), z(t-r), w(t-r), u(t-r))-\alpha_{1}\right] u^{2} \\
& +\frac{\alpha_{4}\left(\alpha_{1} \alpha_{2}-\alpha_{3}\right)}{\alpha_{1} \alpha_{4}-\alpha_{5}}\left[f_{1}(t, x(t-r), y(t-r), z(t-r), w(t-r), u(t-r))-\alpha_{1}\right] z u \\
& +\frac{\varepsilon \alpha_{2}}{16} z^{2}
\end{aligned}
$$




$$
\begin{aligned}
V_{5}= & \frac{1}{4}\left[f_{1}(t, x(t-r), y(t-r), z(t-r), w(t-r), u(t-r))-\alpha_{1}\right] u^{2} \\
& +\alpha_{1}\left[f_{1}(t, x(t-r), y(t-r), z(t-r), w(t-r), u(t-r))-\alpha_{1}\right] w u \\
& +\frac{\varepsilon}{4} w^{2}, \\
V_{6}= & \frac{1}{4}\left[f_{1}(t, x(t-r), y(t-r), z(t-r), w(t-r), u(t-r))-\alpha_{1}\right] u^{2} \\
& +\delta\left[f_{1}(t, x(t-r), y(t-r), z(t-r), w(t-r), u(t-r))-\alpha_{1}\right] y u \\
& +\frac{\varepsilon \alpha_{4}}{4} y^{2}, \\
V_{7}= & \frac{\varepsilon}{4} w^{2}-\left[f_{5}^{\prime}(x)-\alpha_{5}\right] y w+\frac{\varepsilon \alpha_{4}}{4} y^{2}, \\
V_{8}= & \frac{\varepsilon \alpha_{2}}{16} z^{2}-\alpha_{1}\left[f_{5}^{\prime}(x)-\alpha_{5}\right] y z+\frac{\varepsilon \alpha_{4}}{4} y^{2} .
\end{aligned}
$$

Now, subject to the assumptions (ii) and (iii) of theorem, one easily finds that

$$
V_{4} \geq 0, \quad V_{5} \geq 0, \quad V_{6} \geq 0, \quad V_{7} \geq 0, \quad V_{8} \geq 0,
$$

respectively. Gathering the above discussion into (8) and making use of the assumption (ii), it follows that

$$
\begin{aligned}
\dot{V}_{0} \leq & -\frac{1}{4}\left[\varepsilon_{0}-2 \alpha_{5} r\right] u^{2}-\frac{1}{4}\left[2 \varepsilon-\left(4 \rho+2 \alpha_{1} \alpha_{5}\right) r\right] w^{2} \\
& -\left[\frac{7 \varepsilon \alpha_{2}}{8}-\left(\mu+\frac{\alpha_{4} \alpha_{5}\left(\alpha_{1} \alpha_{2}-\alpha_{3}\right)}{2\left(\alpha_{1} \alpha_{4}-\alpha_{5}\right)}\right) r\right] z^{2}-\left[\frac{\varepsilon \alpha_{4}}{4}-\left(\frac{\delta \alpha_{5}}{2}+\lambda\right) r\right] y^{2} \\
& +\left[\frac{\alpha_{4} \alpha_{5}\left(\alpha_{1} \alpha_{2}-\alpha_{3}\right)}{2\left(\alpha_{1} \alpha_{4}-\alpha_{5}\right)}+\frac{\alpha_{5}}{2}+\frac{\alpha_{1} \alpha_{5}}{2}+\frac{\delta \alpha_{5}}{2}-\lambda\right] \int_{t-r}^{t} y^{2}(s) d s \\
& +\left|u+\alpha_{1} w+\frac{\alpha_{4}\left(\alpha_{1} \alpha_{2}-\alpha_{3}\right)}{\alpha_{1} \alpha_{4}-\alpha_{5}} z+\delta y\right| \\
& \times|p(t, x(t-r), y(t-r), z(t-r), w(t-r), u(t-r))| \\
& -\mu \int_{t-r}^{t} z^{2}(s) d s-\rho \int_{t-r}^{t} w^{2}(s) d s .
\end{aligned}
$$


If we let

$$
\begin{gathered}
\lambda=\left[\frac{\alpha_{4} \alpha_{5}\left(\alpha_{1} \alpha_{2}-\alpha_{3}\right)}{2\left(\alpha_{1} \alpha_{4}-\alpha_{5}\right)}+\frac{\alpha_{5}}{2}+\frac{\alpha_{1} \alpha_{5}}{2}+\frac{\delta \alpha_{5}}{2}\right], \\
\mu=\frac{\alpha_{4} \alpha_{5}\left(\alpha_{1} \alpha_{2}-\alpha_{3}\right)}{2\left(\alpha_{1} \alpha_{4}-\alpha_{5}\right)} \quad \text { and } \rho=\frac{\alpha_{1} \alpha_{5}}{2},
\end{gathered}
$$

then subject to the assumption (i) of theorem the inequality (9) implies that

$$
\begin{aligned}
\dot{V}_{0} \leq & -\frac{1}{4}\left[\varepsilon_{0}-2 \alpha_{5} r\right] u^{2}-\frac{1}{4}\left[2 \varepsilon-4\left(\alpha_{1} \alpha_{5}\right) r\right] w^{2} \\
& -\left[\frac{7 \varepsilon \alpha_{2}}{8}-\frac{\alpha_{4} \alpha_{5}\left(\alpha_{1} \alpha_{2}-\alpha_{3}\right)}{\alpha_{1} \alpha_{4}-\alpha_{5}} r\right] z^{2}-\left[\frac{\varepsilon \alpha_{4}}{4}-\left(\frac{\delta \alpha_{5}}{2}+\lambda\right) r\right] y^{2} \\
& +\left|u+\alpha_{1} w+\frac{\alpha_{4}\left(\alpha_{1} \alpha_{2}-\alpha_{3}\right)}{\alpha_{1} \alpha_{4}-\alpha_{5}} z+\delta y\right| \\
& \times|p(t, x(t-r), y(t-r), z(t-r), w(t-r), u(t-r))|
\end{aligned}
$$

Thus, one easily obtains that

$$
\begin{aligned}
& \frac{d}{d t} V_{0}\left(t, x_{t}, y_{t}, z_{t}, w_{t}, u_{t}\right) \leq-\tau\left(y^{2}+z^{2}+w^{2}+u^{2}\right) \\
& \quad+\left|u+\alpha_{1} w+\frac{\alpha_{4}\left(\alpha_{1} \alpha_{2}-\alpha_{3}\right)}{\alpha_{1} \alpha_{4}-\alpha_{5}} z+\delta y\right| \\
& \quad \times|p(t, x(t-r), y(t-r), z(t-r), w(t-r), u(t-r))|
\end{aligned}
$$

for some constant $\tau>0$ provided that

$$
r<\min \left\{\frac{\varepsilon_{0}}{2 \alpha_{5}}, \frac{\varepsilon}{2 \alpha_{1} \alpha_{5}}, \frac{7 \varepsilon \alpha_{2}\left(\alpha_{1} \alpha_{4}-\alpha_{5}\right)}{8 \alpha_{4} \alpha_{5}\left(\alpha_{1} \alpha_{2}-\alpha_{3}\right)}, \frac{\varepsilon \alpha_{4}}{2\left(\delta \alpha_{5}+2 \lambda\right)}\right\} .
$$

Clearly, we have

$$
\begin{aligned}
& \frac{d}{d t} V_{0}\left(t, x_{t}, y_{t}, z_{t}, w_{t}, u_{t}\right) \leq\left|u+\alpha_{1} w+\frac{\alpha_{4}\left(\alpha_{1} \alpha_{2}-\alpha_{3}\right)}{\alpha_{1} \alpha_{4}-\alpha_{5}} z+\delta y\right| \\
& \quad \times|p(t, x(t-r), y(t-r), z(t-r), w(t-r), u(t-r))| \\
& \quad \leq\left(|u|+\alpha_{1}|w|+\frac{\alpha_{4}\left(\alpha_{1} \alpha_{2}-\alpha_{3}\right)}{\alpha_{1} \alpha_{4}-\alpha_{5}}|z|+\delta|y|\right) q(t) \\
& \quad \leq D_{7}(|y|+|z|+|w|+|u|) q(t),
\end{aligned}
$$


where

$$
D_{7}=\max \left\{1, \alpha_{2}, \frac{\alpha_{4}\left(\alpha_{1} \alpha_{2}-\alpha_{3}\right)}{\alpha_{1} \alpha_{4}-\alpha_{5}}, \delta\right\}
$$

Using the fact $|y|<1+y^{2},|z|<1+z^{2},|w|<1+w^{2}$ and $|u|<1+u^{2}$, we see that

$$
\frac{d}{d t} V_{0}\left(t, x_{t}, y_{t}, z_{t}, w_{t}, u_{t}\right) \leq D_{7}\left[4+\left(y^{2}+z^{2}+w^{2}+u^{2}\right)\right] q(t) .
$$

In view of (7), it follows that

$$
\frac{d}{d t} V_{0}\left(t, x_{t}, y_{t}, z_{t}, w_{t}, u_{t}\right) \leq 4 D_{7} q(t)+D_{7} D_{8}^{-1} V_{0}\left(t, x_{t}, y_{t}, z_{t}, w_{t}, u_{t}\right) q(t) .
$$

Finally, integrating this inequality from $t_{0},\left(t_{0} \geq 0\right)$, to $t$ and using the assumption $q \in L^{1}(0, \infty)$ and Gronwall-Reid-Bellman inequality, we conclude that

$$
\begin{aligned}
& V_{0}\left(t, x_{t}, y_{t}, z_{t}, w_{t}, u_{t}\right) \leq V_{0}\left(t_{0}, x_{t_{0}}, y_{t_{0}}, z_{t_{0}}, w_{t_{0}}, u_{t_{0}}\right) \\
& \quad+4 D_{7} A+D_{7} D_{8}^{-1} \int_{t_{0}}^{t}\left(V_{0}\left(s, x_{s}, y_{s}, z_{s}, w_{s}, u_{s}\right)\right) q(s) d s \\
& \quad \leq\left[V_{0}\left(t_{0}, x_{t_{0}}, y_{t_{0}}, z_{t_{0}}, w_{t_{0}}, u_{t_{0}}\right)+4 D_{7} A\right] \exp \left(D_{7} D_{8}^{-1} \int_{t_{0}}^{t} q(s) d s\right) \\
& \quad \leq\left[V_{0}\left(t_{0}, x_{t_{0}}, y_{t_{0}}, z_{t_{0}}, w_{t_{0}}, u_{t_{0}}\right)+4 D_{7} A\right] \exp \left(D_{7} D_{8}^{-1} A\right)=K_{1}<\infty,
\end{aligned}
$$

where $K_{1}>0$ is a constant,

$$
K_{1}=\left[V_{0}\left(t_{0}, x_{t_{0}}, y_{t_{0}}, z_{t_{0}}, w_{t_{0}}, u_{t_{0}}\right)+4 D_{7} A\right] \times \exp \left(D_{7} D_{8}^{-1} A\right)
$$

and

$$
A=\int_{0}^{\infty} q(s) d s .
$$

Now, the inequality (7) and the last inequality together give that

$$
x^{2}+y^{2}+z^{2}+w^{2}+u^{2} \leq D_{8}^{-1} V\left(t, x_{t}, y_{t}, z_{t}, w_{t}, u_{t}\right) \leq K,
$$

where $K=K_{1} D_{8}^{-1}$. This fact completes the proof of theorem. 


\section{REFERENCES}

[1] A.M.A. Abou-El-Ela and A.I. Sadek, On the boundedness and periodicity of a certain differential equation of fifth order. Z. Anal. Anwendungen, 11(2) (1992), 237-244.

[2] T.A. Burton, Stability and Periodic Solutions of Ordinary and Functional Differential Equations. Academic Press, Orlando (1985).

[3] E.N. Chukwu, On the boundedness and stability of solutions of some differential equations of the fifth order. SIAM J. Math. Anal., 7(2) (1976), 176-194.

[4] L.È. Èl'sgol'ts, Introduction to the theory of differential equations with deviating arguments. Translated from the Russian by Robert J. McLaughlin Holden-Day, Inc., San Francisco, Calif.-London-Amsterdam (1966).

[5] L.È. Èl'sgol'ts and S.B. Norkin, Introduction to the theory and application of differential equations with deviating arguments. Translated from the Russian by John L. Casti. Mathematics in Science and Engineering, Vol. 105. Academic Press [A Subsidiary of Harcourt Brace Jovanovich, Publishers], New York-London (1973).

[6] K. Gopalsamy, Stability and oscillations in delay differential equations of population dynamics. Mathematics and its Applications, 74. Kluwer Academic Publishers Group, Dordrecht (1992).

[7] J. Hale, Theory of Functional Differential Equations. Springer-Verlag, New York-Heidelberg (1977).

[8] J. Hale and S.M. Verduyn Lunel, Introduction to functional-differential equations. Applied Mathematical Sciences, 99. Springer-Verlag, New York (1993).

[9] V. Kolmanovskii and A. Myshkis, Introduction to the Theory and Applications of Functional Differential Equations. Kluwer Academic Publishers, Dordrecht (1999).

[10] V.B. Kolmanovskii and V.R. Nosov, Stability of functional-differential equations. Mathematics in Science and Engineering, 180. Academic Press, Inc. [Harcourt Brace Jovanovich, Publishers], London (1986).

[11] N.N. Krasovskii, Stability of motion. Applications of Lyapunov's second method to differential systems and equations with delay. Translated by J.L. Brenner Stanford University Press, Stanford, Calif. (1963).

[12] A.M. Liapunov, Stability of Motion. Academic Press, London (1966).

[13] G. Makay, On the asymptotic stability of the solutions of functional-differential equations with infinite delay. J. Differential Equations, 108(1) (1994), 139-151.

[14] A.S.C. Sinha, On stability of a fifth-order nonlinear differential equation. Proceedings of the IEEE, September (1971), 1382-1383.

[15] C. Tunç, On the boundedness and the stability results for the solutions of certain fifth order differential equations. Istanbul Üniv. Fen Fak. Mat. Derg., 54 (1995), 151-160 (1997). 
[16] C. Tunç, On the boundedness and the stability results for the solution of certain fifth order differential equations. Ann. Differential Equations, 12(3) (1996), 259-266.

[17] C. Tunç, A study of the stability and boundedness of the solutions of nonlinear differential equations of the fifth order. Indian J. Pure Appl. Math., 33(4) (2002), 519-529.

[18] C. Tunç, On the boundedness of solutions of nonlinear differential equations of fifthorder with delay. Proceedings of Dynamic Systems and Applications 1, 5 (2008), 466-473.

[19] C. Tunç, A theorem on the boundedness of solutions to fifth-order nonlinear differential equations with delay. Annales des Sciences Mathématiques du Québec, (2008), (accepted for publication).

[20] T. Yoshizawa, Stability theory by Liapunov's second method. The Mathematical Society of Japan, Tokyo (1966).

[21] Y. Yuan Hong, Stability and boundedness of solutions to nonlinear differential equations of the fifth order. (Chinese) J. Central China Normal Univ. Natur. Sci., 24(3) (1990), 267-273. 